\title{
High phenylalanine levels directly affect mood and sustained attention in adults with phenylketonuria: a randomised, double-blind, placebo-controlled, crossover trial
}

\author{
Amber E. ten Hoedt • Leo M. J. de Sonneville • Baudouin Francois • \\ Nienke M. ter Horst • Mirian C. H. Janssen • M. Estela Rubio-Gozalbo • \\ Frits A. Wijburg • Carla E. M. Hollak • Annet M. Bosch
}

Received: 9 September 2010 /Revised: 9 November 2010 /Accepted: 17 November 2010 / Published online: 10 December 2010

(C) The Author(s) 2010. This article is published with open access at Springerlink.com

\begin{abstract}
The main debate in the treatment of Phenylketonuria (PKU) is whether adult patients need the strict phenylalanine (Phe)-restricted diet. Physicians and patients lack evidence-based guidelines to help them make well-informed choices. We have carried out the first randomised double-blind placebo-controlled trial into the effects of short-term elevation of Phe levels on
\end{abstract}

Communicated by: Nenad Blau

A. E. ten Hoedt $\cdot$ N. M. ter Horst · F. A. Wijburg •

A. M. Bosch $(\triangle)$

Department of Pediatrics (H7-270), Academic Medical Centre, University of Amsterdam,

Meibergdreef 9 ,

1105 AZ, Amsterdam, The Netherlands

e-mail: a.m.bosch@amc.uva.nl

L. M. J. de Sonneville

Leiden Institute for Brain and Cognition, Leiden University,

Leiden, The Netherlands

B. Francois

Department of Metabolic Diseases, Centrum Pinocchio,

Diepenbeek, Belgium

\section{C. H. Janssen}

Department of Internal Medicine,

Radboud University Medical Centre,

Nijmegen, The Netherlands

M. E. Rubio-Gozalbo

Department of Pediatrics and Laboratory Genetic Metabolic Diseases, Maastricht University Medical Centre,

Maastricht, The Netherlands

C. E. M. Hollak

Department of Internal Medicine, Academic Medical Centre, University of Amsterdam,

Amsterdam, The Netherlands neuropsychological functions and mood of adults with PKU. Nine continuously treated adults with PKU underwent two 4-week supplementation periods: one with Phe, mimicking normal dietary intake, and one with placebo in randomly allocated order via a randomisation coding list in a double-blind cross-over design. A set of neuropsychological tests (Amsterdam Neuropsychological Tasks) was administered at the end of each study period. In addition, patients and for each patient a friend or relative, completed weekly Profile of Mood States (POMS) questionnaires, evaluating the patients' mood. Phe levels were measured twice weekly. Mean plasma Phe levels were significantly higher during Phe supplementation compared with placebo $(p=0.008)$. Neuropsychological tests demonstrated an impairment in sustained attention during Phe supplementation ( $p=$ 0.029). Both patients and their friend or relative reported lower scores on the POMS questionnaires during Phe supplementation ( $p=0.017$ and $p=0.040$, respectively). High plasma Phe levels have a direct negative effect on both sustained attention and on mood in adult patients with PKU. A Phe-restricted "diet for life" might be an advisable option for many.

\section{Introduction}

Phenylketonuria (PKU; OMIM 261600) is an autosomal recessive inborn error of metabolism caused by a deficiency of the enzyme phenylalanine hydroxylase (PAH; EC 1.14.16.1), which catalyses the conversion of the amino acid phenylalanine (Phe) into tyrosine. PKU is a pan-ethnic disease with an estimated birth prevalence varying from 1:2,500 to $1: 125,000$ (Scriver et al. 2008), and it has been diagnosed in approximately 13,000 people 
in the U.S. and 50,000 people worldwide. The enzyme deficiency results in a high concentration of Phe which is neurotoxic, particularly during the first years of life (Albrecht et al. 2009). Untreated PKU results in severe intellectual disability and neurological sequelae (Scriver et al. 2008). Since the 1960s, these complications can be prevented by detection through newborn screening followed by dietary treatment consisting of a restriction of Phe intake by a protein-restricted diet and supplementation of all amino acids but Phe. However, a highly controversial issue in the treatment of PKU is whether a strict dietary control of Phe levels is still necessary in adults. With the first PKU patients detected by newborn screening now in their $40 \mathrm{~s}$, there is a rapidly growing cohort of adults with early and continuously treated PKU for whom evidence-based treatment guidelines are lacking. Current dietary guidelines for adults vary widely (Van Spronsen et al. 2009), and in some countries relaxation of the diet in adolescence is common practice (SchweitzerKrantz and Burgard 2000). Dietary relaxation for adults might be considered safe on the basis of the observation that discontinuation of the diet does not cause a major decrease in IQ in adults (Burgard 2000). However, mild cognitive sequelae including executive function deficits have been reported (Christ et al. 2010a). These deficits, associated with concurrent high Phe levels, have been observed in both children and adults (Huijbregts et al. 2002b, c; Schmidt et al. 1994). In other disorders, impairments in executive function have been correlated with deficits in social relationships and communication skills (Beauchamp and Anderson 2010). Self reports by PKU patients as well as experiences of treating physicians, including ourselves, suggest that high Phe levels may in some cases result in irritability, impacting on adaptive social skills (www.pku.com, www.childrensmemorial.org/ depts/genetics/pku.aspx) However, the effect of high Phe levels on mood in continuously treated adults with PKU has never been studied.

In order to improve guidelines on dietary therapy for adults with PKU, the consequences of elevated Phe levels mimicking a full relaxation of the diet need to be studied prospectively. To this end, we performed a double-blind placebo-controlled crossover Phe-loading study in early and continuously treated adults with PKU focusing on the impact of high Phe levels on executive functioning as well as on mood.

\section{Patients and methods}

Patients

Patients were recruited via treating physicians in the Academic Medical Center Amsterdam (AMC), the Univer- sity Medical Centres of Groningen, Maastricht, and Nijmegen (The Netherlands), and the Centrum Pinocchio, Diepenbeek (Belgium). Patients were enrolled in the study between December 2007 and December 2008. Patients with PKU aged $\geq 18$ years, who were diagnosed by newborn screening and continuously treated and had a mean plasma Phe level $<1,100 \mu \mathrm{mol} / \mathrm{L}$ in the year prior to the start of the study, were eligible for inclusion. Exclusion criteria included mental or legal incapacitation, drug or alcohol misuse, a diagnosis of neurological disease, psychiatric illness, lack of fluency in Dutch and inability to comply with study procedures. Additional exclusion criteria for females were pregnancy or the wish to become pregnant within a year of the start of the study. Patients signed an institutionally approved informed consent prior to enrolment. Approval for this study was granted by the Ethical Committee of the AMC, Amsterdam. The trial was registered with The Netherlands National Trial Register (NTR \# 1056) before recruiting of patients.

\section{Procedure}

Patients were instructed to continue their prescribed natural protein-restricted diet and supplementation of amino acids throughout the study. To determine intake of Phe, daily consumption of natural protein was evaluated in a 3-day dietary record by a dietician prior to the start of the study. The trial had a double-blind cross-over design. Patients were randomly assigned to one of the two groups, starting either with placebo-containing capsules or with Phe-containing capsules. After 4 weeks, patients crossed over to the alternative treatment arm.

During the period in which Phe capsules were consumed, each patient received an individually calculated amount of Phe, taking into account their dietary Phe intake, to reach the total Phe intake likely to be consumed by a healthy adult of the same sex and weight and thus mimicking the condition of being fully 'off diet'. The protein intake of healthy Dutch adults is higher in men than in women. Average Phe intakes aimed at were: $4,500 \mathrm{mg}$ per day (mpd) for male patients between 60 and $\leq 95 \mathrm{~kg}$, 4,000 mpd $<60 \mathrm{~kg}, 5,000 \mathrm{mpd}>95 \mathrm{~kg}, 3,000 \mathrm{mpd}$ for female patients between 50 and $\leq 85 \mathrm{~kg}, 2,500 \mathrm{mpd}<50 \mathrm{~kg}$, and $3,500 \mathrm{mpd}>85 \mathrm{~kg}$.

For placebo and Phe, capsules were identical in appearance. Patients had the choice to swallow the capsules whole or to open the capsules and add the content to their amino acid mixture. Patients took 2-8 capsules a day (average 5 capsules), divided over 3 doses. To monitor the compliance with the capsules, patients recorded the intake of the capsules in a diary. Between the two study periods, there was a washout period of at least 4 weeks in which 
patients consumed their usual PKU diet and amino acid formulas.

\section{Randomisation and masking}

An independent data manager rendered a randomisation coding list. A non-deterministic version of the minimisation method (Pocock and Simon 1975) was used to balance treatment arms. No stratification factors were used. This list, which contained numbers linked to the patients by the study physician, and allocated treatment assignments, was sent to the pharmaceutical company which used it to prepare the capsules. All patients and investigators were masked to treatment assignment.

Capsules were prepared and coded by Nutricia Liverpool (Liverpool, UK) and distributed to the patients by the study physician (A.H.) at the start of each study period. Capsules were packed in identical tubs, labelled with patient number, phase number, and a three-letter code and barcode indicating placebo or Phe. In order to prevent identification and comparison by the patients, three different three-letter codes were used for both Phe- and placebo-containing capsules.

\section{Biochemical monitoring}

Phe levels in bloodspots were measured twice weekly during the two 4-week study periods and during the week before each study period. This was achieved using tandem mass spectrometry (standard neutral loss method). Results were not disclosed to the investigators until data were ready for analysis.

\section{Neuropsychological measures}

The primary outcome measure was the change in neuropsychological functioning between the placebo and the Phe supplementation periods.

The Amsterdam Neuropsychological Tasks (ANT) program (De Sonneville 2005, 2009) was used to evaluate neuropsychological function. ANT has successfully been used to determine neurocognitive deficit profiles for various clinical conditions, including PKU (Burgard et al. 1997; Huijbregts et al. 2002b). Various ANT paradigms have been shown to be sensitive to changes in neuropsychological functioning following dietary interventions in children (Huijbregts et al. 2002b) and adults (Schmidt et al. 1994) with PKU. These tasks were selected to draw out a neuropsychological profile associated with Phe-loading. Measures included simple reaction time, sustained attention, visuospatial processing, working memory capacity, inhibition and set shifting and visuomotor control (random pursuit, circle tracing). Detailed task descriptions can be found elsewhere (De Sonneville et al. 2002; Pietz et al. 1993). Based on earlier studies (Huijbregts et al. 2003; Pietz et al. 1993), Phe-loading was expected to unfavourably affect task performance on all tasks except on simple reaction time and simple visuomotor control (circle tracing). Neuropsychological assessment was performed four times: twice before the first study period in order to minimise learning effects which may occur after repeated administration of neuropsychological tests and on the last day of each 4-week study period. The results of the latter two assessments were subjected to statistical analysis.

Tasks were administered using a computer. The input device for the patients' responses was a standard mouse with symmetrical buttons. Prior to task administration, patients practised until the tasks were understood.

IQ was estimated using a 4-subtests short form of the Wechsler Adult Intelligence Scale-Revised (WAIS-III; Wechsler 1981) consisting of vocabulary, picture completion, block design and similarities.

In all patients, neuropsychological tests and IQ tests were performed by one investigator (A.H.).

\section{Clinical measures}

The secondary outcome measure was the change in mood state of the patients, which was evaluated by the standardised "Profile of Mood States" (POMSr) questionnaire (revised version) (Wald and Mellenbergh 1990). This questionnaire is designed to provide data on five categories of mood states: tension, depression, anger, vigour, fatigue (mood types). The questionnaire was completed weekly during each 4-week study period by the patients and by a significant other of each patient (spouse, friend or close colleague) reporting on the patient's mood.

\section{Statistical analysis}

Task performance data of the ANT, obtained at the end of the two study periods, were pooled across groups per condition (placebo, Phe). Repeated measures analysis of variance (ANOVA), with experimental condition (placebo vs Pheloading) as within-subject factor was used to test changes in neuropsychological outcome. Additional within-subject factors, when relevant, were entered to test the interactions of intervention with task manipulations (for example: increase in memory load, in visuospatial complexity, etc.). Separate runs were made with reaction time and number of errors as a dependent variable.

Similarly, scale scores for the five categories of mood states of the POMSr were computed per type of observer (patient and friend or relative) and the results were pooled across groups per experimental condition. Before statistical analysis, an average score of all patients on the five 
different categories of mood state was calculated, for both study periods. To test whether mood differed between treatment conditions and whether differences were mood type-dependent, scores were subjected to repeated measures analyses ANOVA with experimental condition and mood type as within-subject factor. Separate runs were made for each observer type. Effect sizes were estimated by means of partial eta squared $\left(\eta_{p}{ }^{2}\right)$. Large effects correspond with $\eta_{p}{ }^{2} \geq 0.14$, moderate effects with $\eta_{p}{ }^{2} \geq 0.06$ and $\eta_{p}{ }^{2}<0.14$, and weak effects with $\eta_{p}{ }^{2}<0.06$ (Cohen 1988).

Prior to analysis, the vigour scale scores have been recoded to the effect that higher scores are associated with an unfavourable outcome (less vigour), in the same way that higher scores denote more tension, more depression, more anger and more fatigue. For the analysis of the effect of the loading condition on mood and the analysis of the neuropsychological task result, alpha was set at 0.05. Posthoc power analyses was applied to all trend and nonsignificant results that were interpreted as meaningful on the basis of the estimated effect sizes.

\section{Results}

\section{Patients}

On the basis of inclusion and exclusion criteria and expected willingness to participate at the discretion of the treating physician, 129 patients were eligible to participate in this study. Of them, 12 patients agreed to participate. Fear of effects of elevated Phe levels, the time demanded by the study and the fact that most patients had already participated in multiple previous studies on PKU were the most prominent reasons for not participating. One patient was excluded before the start of the study because of a co-existing illness possibly affecting cognition. Between December 2007 and December 2008, patients were randomised; the study was completed in April 2009. Patients were randomly allocated to one of the treatment arms: either
Phe-loading-placebo or placebo-Phe-loading. One patient withdrew before the start of the investigations because of pressure at work and one patient withdrew after the first neuropsychological test because his partner feared elevatedPhe-related negative changes in his behaviour. All the remaining 9 patients completed the study. Table 1 shows the patients' baseline characteristics, including pre-study dietary Phe intake and mean blood Phe value in the year prior to the study, reflecting a very variable individual Phe tolerance in this cohort that includes classical as well as mild PKU patients. Compliance with taking the capsules was $99.6 \%$ in the first and $99.1 \%$ in the second study period.

\section{IQ}

WAIS IQs, estimated from the 4-subtest short form of the Wechsler Adult Intelligence Scale-Revised were within normal range ( $M=97.22$, range 83-117), (Table 1), although slightly below the population mean, which may be due to sampling error in this small sample size but is also consistent with previous studies (Pietz et al. 1998; Schmidt et al. 1996).

Phe levels

Mean (standard deviation) blood Phe value was $1,259 \mu \mathrm{mol} / \mathrm{L}( \pm 332)$ in the Phe-loading phase and $709 \mu \mathrm{mol} / \mathrm{L}( \pm 322)$ in the placebo phase, which was significantly different (Wilcoxon signed-ranks test $p=$ 0.008) (Fig. 1). In the week prior to the second study period, after a washout of at least 4 weeks, the mean Phe level was $702 \mathrm{umol} / \mathrm{L}( \pm 389)$, which is comparable to the mean Phe level in the week prior to the first study period of $790 \mathrm{umol} / \mathrm{L}( \pm 332)$.

\section{Mood}

Figure 2 shows the mean scale scores per observer (self, friend or relative) on the different subscales of the POMSr during Phe-loading or placebo. Self-reported

Table 1 Baseline characteristics

\begin{tabular}{lllllll}
\hline Patient & Gender & Age (years) & Weight $(\mathrm{kg})$ & IQ & $\begin{array}{l}\text { Total pre-study Phe } \\
\text { intake }(\mathrm{mg})\end{array}$ & $\begin{array}{l}\text { Mean pre-study blood } \\
\text { Phe value }(\mu \mathrm{mol} / \mathrm{L})\end{array}$ \\
\hline 1 & F & 20 & 65 & 105 & 2,350 & 560 \\
2 & M & 28 & 73 & 83 & 1,175 & 784 \\
3 & F & 26 & 80 & 102 & 1,750 & 427 \\
4 & F & 23 & 50 & 87 & 1,595 & 978 \\
5 & M & 34 & 75 & 105 & 1,470 & 936 \\
6 & F & 20 & 60 & 117 & 1,095 & 346 \\
7 & F & 22 & 67 & 83 & 580 & 635 \\
8 & F & 19 & 78 & 86 & 535 & 388 \\
9 & M & 20 & 94 & 107 & 810 & 786 \\
\hline
\end{tabular}




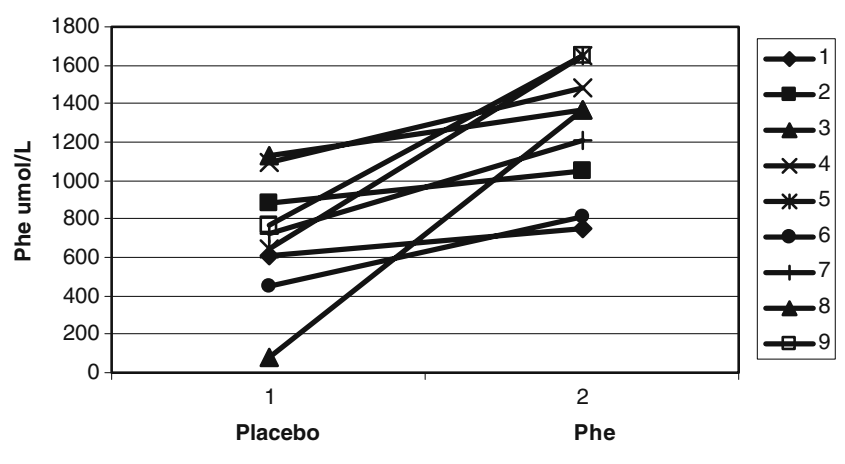

Fig. 1 Mean Phe levels per patient

mood states were significantly less favourable during the Pheloading period compared to the placebo period $\left(F_{1,8}=9.03\right.$, $\left.p=0.017, \eta_{\mathrm{p}}{ }^{2}=0.53\right)$. Post-hoc testing revealed patients to feel more depressed (trend, $p=0.097, \eta_{p}{ }^{2}=0.31$, power= 0.38 ), more fatigued $\left(p=0.021, \eta_{p}{ }^{2}=0.51\right)$, and less vigorous $\left(p=0.006, \eta_{p}{ }^{2}=0.64\right)$. Similarities were found for the report by a friend or relative. Overall mood was significantly less favourable during Phe-loading $\left(F_{1,8}=6.02, p=0.040, \eta_{p}{ }^{2}=\right.$ 0.43 , power $=0.58]$ and post-hoc testing showed that the friend or relative reported more depression (trend, $p=0.057$, $\eta_{p}{ }^{2}=0.38$, power $=0.50$ ) and more fatigue (trend, $p=0.069$, $\eta_{p}{ }^{2}=0.36$, power $\left.=0.46\right)$ as well. Differences were that the friend or relative reported more anger (trend, $p=0.052, \eta_{p}{ }^{2}=$ 0.39 , power $=0.52$ ) and did not report a less vigorous mood state. One friend or relative lost the questionnaires completed during the placebo phase. The missing scale scores for this patient were substituted by the mean scale scores, obtained during the placebo phase, of the remaining group of the friends or relatives.

\section{Neuropsychological profile}

Statistical analysis of the neuropsychological performance data showed that Phe-loading resulted in a significant larger fluctuation in tempo during sustained attention compared to the placebo supplementation $\left(F_{1,8}=7.02, p=0.029, \eta_{p}{ }^{2}=\right.$ 0.47 , power $=0.64)$. On all other measures of neuropsychological function, the effects of Phe-loading were not significant, although differences on two following tasks were accompanied by large effect sizes: Phe-loading resulted in slower reaction times $\left(\eta_{p}{ }^{2}=0.20\right.$, power $\left.=0.25\right)$ in the inhibition and set shifting task and in slower recognition of complex patterns $\left(\eta_{p}{ }^{2}=0.16\right)$ in the visuospatial pattern recognition task.

\section{Discussion}

This is the first double-blind, randomised, placebocontrolled cross over trial designed to investigate the effects of a fully normalised Phe intake on both executive function and mood in adults with PKU.

We demonstrated that elevated Phe levels are associated with a negative effect on sustained attention as well as on the mood of the patients. Indeed, the high Phe levels impaired sustained attention by increasing the fluctuation in speed, which has been previously reported (Huijbregts et al. 2002c; Schmidt et al. 1994). Hypothetically, the impairment of sustained attention might have consequences for the daily functioning of patients with PKU. Insufficient attention to tasks can result in 'slips' of action or everyday attention failures as automatic unintended action sequences are triggered inappropriately. Such slips can arise from deficits in sustained attention which have been reported to occur following frontal lobe and white matter damage from very different causes (e.g. in traumatic brain injury) (Robertson et al. 1997).

It is therefore interesting that white matter changes have been demonstrated in patients with treated PKU (Pietz et al. 1998; Vermathen et al. 2007) and that the prefrontal cortex is thought to play a role in the pathophysiology of the neurological complications in PKU (Welsh 1996; Christ et al. 2010b). Joseph and Dyer (2003) reported dopamine depletion in the prefrontal cortex of a PKU mouse model, which was thought to be caused by hypomyelinisation leading to down-regulation of neurotransmitter production (Joseph and Dyer 2003). Another cause for dopamine depletion in patients with PKU may be the low levels of its precursor tyrosine, caused by competition of high Phe levels with the uptake of tyrosine across the blood-brain barrier as both amino acids are transported by the same neutral amino acid transporter (Welsh 1996). Dopaminergic neurons that project on the prefrontal cortex are highly sensitive to even small decreases in tyrosine (Tam and Roth 1997), and dopamine is believed to be involved in frontal lobe functions, such as working memory, information processing and attention (Nieoullon and Coquerel 2003).

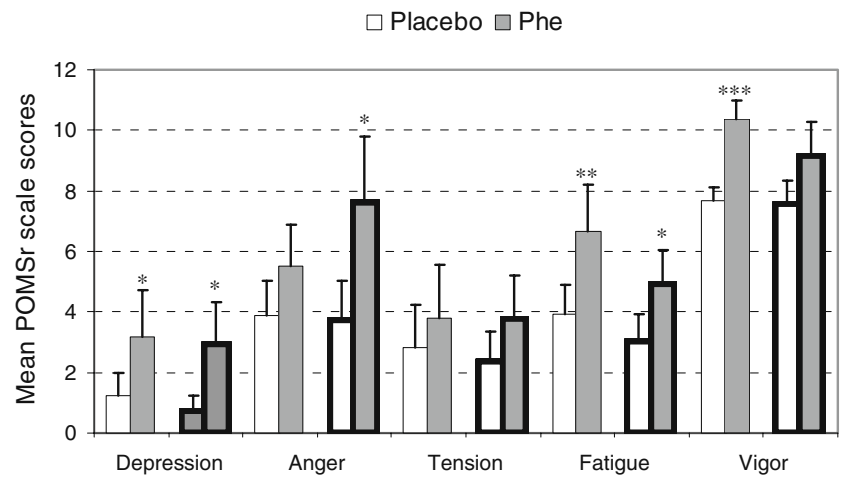

Fig. 2 Mean $\pm \mathrm{SE}$ of POMSr scale scores as function of treatment, mood type and observer. Patient self report $(-)$, Significant other report (一): ${ }^{* * *} p \leq 0.01,{ }^{* *} p \leq 0.05,{ }^{*} p<0.10$ 
We observed no impairment in baseline speed, pursuit or tracking, which is consistent with reports in the literature (Huijbregts et al. 2003). PKU patients have deficits in tasks requiring a high level of monitoring and manipulation (Diamond et al. 1997; Huijbregts et al. 2002a, b). The pursuit task consists of following a randomly moving object and requires more concurrent planning and adaptation compared to the tracking task. It might be expected for PKU patients to have lower scores on the pursuit task, which we did not find. One explanation might be that the pursuit task and the baseline speed task have a much shorter duration compared to the sustained attention task and are not demanding enough.

In contrast with the other studies, we observed no significant difference in performance on the inhibition, set shifting and memory task (Huijbregts et al. 2002a; Pietz et al. 1993). However, large effect sizes $\left(\eta_{p}{ }^{2} \geq 0.14\right)$ were seen on reaction times in the inhibition and moderate effect sizes on the set shifting task. A large and a moderate effects size was also observed in the tasks on recognition of complex patterns and visuospatial pattern recognition task, respectively, suggesting an influence of high Phe levels.

Importantly, we report for the first time a clear association between high blood Phe levels and poor mood when compared with the placebo supplementation. Crucially, this effect was reported by both patients and their friend or relative, which would suggest that the migration to a more negative mood is sufficiently severe for individuals to be self aware of it.

The possible biochemical nature of this phenomenon could be a lower level of brain serotonin. Like dopamine, tryptophan as a precursor of serotonin competes with Phe for transport across the blood-brain barrier (Hargreaves and Pardridge 1988). Elevated Phe levels might reduce brain tryptophan and consequently serotonin. Reduction in serotonin levels might play a role in the pathogenesis of psychological disorders, including depression, in patients with PKU (Pietz et al. 1997). Thus, the altered mood states due to high Phe levels as observed in our study may also be caused by decreased serotonin levels.

We believe that persistent negative changes in mood may ultimately impact stability in relationships and social interactions and should therefore be avoided. BikMultanowski et al. (2008) demonstrated a hampered quality of life in $45 \%$ of a cohort of patients who were off diet with high Phe levels. Resumption of the diet improved the quality of life of most of these patients, mostly in the domains of anxiety and depressiveness.

We recognise that a limitation of the present study is the small number of participating patients. This may well have prevented unfavourable effects of Phe-loading reaching significance on inhibition and complex visuospatial pattern processing, as these differences showed large effect sizes.
Future studies with a larger cohort of patients are warranted to further explore our findings.

Finally, as only the effects of a relative short period of high Phe levels were studied, we do not know if the observed negative effects would remain in the long term or if adaptive mechanisms may result in a normalisation of mood and performance in patients with PKU who stop following their prescribed diets.

In conclusion, we demonstrated that short-term high Phe levels have a significant direct negative effect on mood and neuropsychological performance in adult patients with PKU. Patients should be educated that potential advantages of going off diet should be balanced against a negative influence on mood, in addition to the consequences of attention failures. A Phe restricted "diet for life" might be an advisable option for many.

Acknowledgments Nutricia Advanced Medical Nutrition provided the funding for this study. The funding source had no role in study design, collection, analysis, and interpretation of data, report writing, or the decision to submit the manuscript for publication. All authors had access to all the data.

Funding Nutricia Advanced Medical Nutrition provided the funding for this study. The funding source had no role in study design, collection, analysis, and interpretation of data, report writing, or the decision to submit the manuscript for publication. All authors had access to all the data.

Competing interest A.B. is a member of a board of experts in metabolic disorders for Nutricia Liverpool and member of PKU advisory boards of Merck Serono Geneva. F.W. is a member of the medical advisory board of Nutricia (the Netherlands) for special products. In addition to his position as associate professor at the University of Leiden, The Netherlands, L.S. is director of the firm (Sonares BV) that commercially distributes the computerised neuropsychological test software (the Amsterdam Neuropsychological Tasks (ANT) program), which has been used in the submitted study. All other authors declare that they have no conflict of interests.

Open Access This article is distributed under the terms of the Creative Commons Attribution Noncommercial License which permits any noncommercial use, distribution, and reproduction in any medium, provided the original author(s) and source are credited.

\section{References}

Albrecht J, Garbade SF, Burgard P (2009) Neuropsychological speed tests and blood phenylalanine levels in patients with phenylketonuria: a meta-analysis. Neurosci Biobehav Rev 33:414-421

Beauchamp MH, Anderson V (2010) SOCIAL: an integrative framework for the development of social skills. Psychol Bull 136:39-64

Bik-Multanowski M, Didycz B, Mozrzymas R et al (2008) Quality of life in noncompliant adults with phenylketonuria after resumption of the diet. J Inherit Metab Dis. Short report \#133 online

Burgard P (2000) Development of intelligence in early treated phenylketonuria. Eur J Pediatr 159:S74-S79 
Burgard P, Rey F, Rupp A, Abadie V, Rey J (1997) Neuropsychologic functions of early treated patients with phenylketonuria, on and off diet: results of a cross-national and cross-sectional study. Pediatr Res 41:368-374

Christ SE, Huijbregts SCJ, de Sonneville LMJ, White DA (2010a) Executive function in early-treated phenylketonuria: profile and underlying mechanisms. Mol Genet Metab 99:S22-S32

Christ SE, Moffitt AJ, Peck D (2010b) Disruption of prefrontal function and connectivity in individuals with phenylketonuria. Mol Genet Metab 99:S33-S340

Cohen J (1988) Statistical power analysis for the behavioral sciences, 2nd edn. Erlbaum, Hillsdale, NJ

De Sonneville LM (2005) Amsterdam neuropsychological tasks: scientific and clinical applications. Tijdschr Neuropsychol 0:27-41

De Sonneville LM (2009) Amsterdam neuropsychological tasks: a computer-aided assessment program. In: Den Brinker BPLM, Beek PJ, Brand AN, Maarse SJ, Mulder LJM (eds) Computers in psychology: cognitive ergonomics, clinical assessment and computer-assisted learning. Swets \& Zeitlinger, Lisse, pp 187-203

De Sonneville LM, Boringa JB, Reuling IE, Lazeron RH, Ader HJ, Polman CH (2002) Information processing characteristics in subtypes of multiple sclerosis. Neuropsychologia 40:1751-1765

Diamond A, Prevor MB, Callender G, Druin DP (1997) Prefrontal cortex cognitive deficits in children treated early and continuously for PKU. Monogr Soc Res Child Dev 62:i-v, 1-208.

Hargreaves KM, Pardridge WM (1988) Neutral amino acid transport at the human blood-brain barrier. J Biol Chem 263:19392-19397

Huijbregts S, de Sonneville L, Licht R, Sergeant J, Van Spronsen F (2002a) Inhibition of prepotent responding and attentional flexibility in treated phenylketonuria. Dev Neuropsychol 22:481-499

Huijbregts SC, De Sonneville LM, Licht R, Van Spronsen FJ, Sergeant JA (2002b) Short-term dietary interventions in children and adolescents with treated phenylketonuria: effects on neuropsychological outcome of a well-controlled population. J Inherit Metab Dis 25:419-430

Huijbregts SC, De Sonneville LM, Licht R, Van Spronsen FJ, Verkerk PH, Sergeant JA (2002c) Sustained attention and inhibition of cognitive interference in treated phenylketonuria: associations with concurrent and lifetime phenylalanine concentrations. Neuropsychologia 40:7-15

Huijbregts SC, De Sonneville LM, Van Spronsen FJ et al (2003) Motor function under lower and higher controlled processing demands in early and continuously treated phenylketonuria. Neuropsychology 17:369-379

Joseph B, Dyer CA (2003) Relationship between myelin production and dopamine synthesis in the PKU mouse brain. J Neurochem 86:615-626

Nieoullon A, Coquerel A (2003) Dopamine: a key regulator to adapt action, emotion, motivation and cognition. Curr Opin Neurol 16:S3-S9
Pietz J, Schmidt E, Matthis P, Kobialka B, Kutscha A, de Sonneville L (1993) EEGs in phenylketonuria. I: Follow-up to adulthood; II: Short-term diet-related changes in EEGs and cognitive function. Dev Med Child Neurol 35:54-64

Pietz J, Fatkenheuer B, Burgard P, Armbruster M, Esser G, Schmidt H (1997) Psychiatric disorders in adult patients with early-treated phenylketonuria. Pediatrics 99:345-350

Pietz J, Dunckelmann R, Rupp A et al (1998) Neurological outcome in adult patients with early-treated phenylketonuria. Eur J Pediatr 157:824-830

Pocock SJ, Simon R (1975) Sequential treatment assignment with balancing for prognostic factors in the controlled clinical trial. Biometrics 31:103-115

Robertson IH, Manly T, Andrade J, Baddeley BT, Yiend J (1997) 'Oops!': performance correlates of everyday attentional failures in traumatic brain injured and normal subjects. Neuropsychologia $35: 747-758$

Schmidt E, Rupp A, Burgard P, Pietz J, Weglage J, de Sonneville L (1994) Sustained attention in adult phenylketonuria: the influence of the concurrent phenylalanine-blood-level. J Clin Exp Neuropsychol $16: 681-688$

Schmidt H, Burgard P, Pietz J, Rupp A (1996) Intelligence and professional career in young adults treated early for phenylketonuria. Eur J Pediatr 155:S97-S100

Schweitzer-Krantz S, Burgard P (2000) Survey of national guidelines for the treatment of phenylketonuria. Eur J Pediatr 159:S70-S73

Scriver R, Levy H, Donlon J (2008) Hyperphenylalaninemia: phenylalanine hydroxylase deficiency. In The Online Metabolic and Molecular Bases of Inherited Disease. Retrieved from: url: www.ommbid.com

Tam SY, Roth RH (1997) Mesoprefrontal dopaminergic neurons: can tyrosine availability influence their functions? Biochem Pharmacol 53:441-453

Van Spronsen FJ, Ahring KK, Gizewska M (2009) PKU-what is daily practice in various centres in Europe? Data from a questionnaire by the scientific advisory committee of the European Society of Phenylketonuria and Allied Disorders. J Inherit Metab Dis 32:58-64

Vermathen P, Robert-Tissot L, Pietz J, Lutz T, Boesch C, Kreis R (2007) Characterization of white matter alterations in phenylketonuria by magnetic resonance relaxometry and diffusion tensor imaging. Magn Reson Med 58:1145-1156

Wald FDM, Mellenbergh GJ (1990) De verkorte versie van de Nederlandse vertaling van de Profile of Mood States (POMS) [The shortened version of the Dutch translation of the Profile of Mood States (POMS)]. Nederlands Tijdschr Psychol 45:86-90

Welsh MC (1996) A prefrontal dysfunction model of early-treated phenylketonuria. Eur J Pediatr 155:S87-S89

Wechsler D (1981) Manual for the Wechsler Adult Intelligence Scale-Revised. Psychological Corporation, New York 